\title{
CONTROLE GLICÊMICO EM PACIENTES DIABÉTICOS TIPO II DURANTE PANDEMIA DE COVID-19
}

\section{Carlos Dalton de Avila Vilanova'; Laureana de Quevedo Barcelos²; Elisângela Colpo $^{3}$}

O Diabetes Mellitus tipo 2 (DM2) é uma doença crônica caracterizada pelo aumento da glicemia, com tratamento baseado no controle glicêmico e manutenção da saúde, no entanto, indivíduos com doenças crônicas sofreram consequências importantes durante a pandemia de Covid-19. O objetivo deste estudo foi revisar dentro da literatura como está o controle glicêmico de pessoas com DM2 na pandemia. Este estudo trata-se de uma revisão integrativa nas bases de dados PubMed, Scielo, Science Direct, foram selecionados artigos com a temática controle glicêmico de pessoas com DM2 durante a pandemia. A maioria dos estudos constataram a deficiência no controle glicêmico, aumento da persepção de estresse e ansiedade devido o menor acesso a serviçoes de saúde, lockdown, redução da prática de atividade física e mudanças alimentares. Sendo possível verificar que a pandemia de Covid-19 provocou impactos no controle glicêmico de pacientes com DM2, aumentando os níveis de hemoglobina glicada (HbA1c).

Palavras-chave: Hemoglobina Glicada, Diabetes Mellitus, Ansiedade, Estresse, SARS-CoV-2.

Eixo Temático: Atenção Integral e Promoção à Saúde (AIPS).

\section{INTRODUÇÃO}

O Diabetes Mellitus (DM) já afetava 463 milhões de pessoas em 2019 no mundo, sendo responsável por 1,5 milhão de mortes em 2016. Estima-se que até 2045, cerca de 700 milhões de pessoas sofrerão de diabetes (SHAW; SICREE; ZIMMET, 2010; INTERNATIONAL DIABETES FEDERATION, 2020).

Com o aumento no número de casos de DM nos países em desenvolvimento, inclusive no Brasil, onde já são mais de 13 milhões de casos, representando 6,9\% dos

\footnotetext{
1 Carlos Dalton de Avila Vilanova - UFN - cdanutri@hotmail.com

2 Laureana de Quevedo Barcelos - UFN - laureanabarcelos@gmail.com

${ }^{3}$ Elisângela Colpo - UFN - elicolpo@ufn.edu.br
} 
brasileiros, o DM se tornou um crescente problema de saúde para todos os países, independente, do seu grau de desenvolvimento. O DM está associado a diversos fatores, dentre eles estão a rápida urbanização, transição epidemiológica e nutricional, estilo de vida sedentário, excesso de peso, envelhecimento populacional crescente, juntamente associada a maior sobrevida dos indivíduos com diabetes (SBD, 2020).

O Diabetes Mellitus tipo 2 (DM2) consiste na redução da eficiência das células $\beta$ pancreáticas e resistência à insulina no músculo, fígado e tecido adiposo, induzindo a consequências metabólicas como secreção de insulina prejudicada, diminuição da captação de glicose hepática e secreção inadequada de glucagon. Também ocorrendo diminuição da produção gastrintestinal de incretinas, com papel fundamental no desenvolvimento da hiperglicemia. Sendo a hiperglicemia o sinal fisiológico do DM2, podendo estar presente antes do início dos sintomas clínicos levando a alterações funcionais em órgãos e tecidos alvo (DEFRONZO, 2009; (RODBARD et al., 2007).

Segundo a American Diabetes Association (2019) o controle glicêmico é a base do tratamento do DM2. No entanto, para ser alcançado e mantido este objetivo é necessária a adoção de hábitos de vida saudáveis que consistem em uma alimentação saudável e prática regular de atividade física, além da adesão ao tratamento farmacológico, quando necessário. Por outro lado, estudos realizados no Brasil e em outros países observaram mudanças no estilo de vida dos adultos em decorrência da pandemia de Covid-19 (GARCIA et al., 2020).

Em dezembro de 2019 foi identificado o vírus SARS-CoV-2, que leva os indivíduos infectados a desenvolverem Covid-19. Devido ao rápido contágio, em 11 de março de 2020 obteve a classificação de pandemia que já se estende por mais de 18 meses. Levando a impactos de saúde em escala mundial, com aproximadamente 100 milhões de pessoas infectadas no primeiro ano, levando a mais de dois milhões de mortes por Covid-19 neste período (ZHU et al., 2020; PARK et al., 2022). Atualmente já são mais de 223 milhões de casos, chegando a 4.6 milhões de mortes por Covid-19 no mundo, destes mais de 20 milhões de casos e 584.421 mortes no Brasil (WHO, 2021).

Em decorrência da rápida evolução da pandemia de Covid-19 foram adotadas 
medidas de controle da disseminação do vírus SARS-CoV-2 em diversos países em todo o mundo, como distanciamento social, que levou a inúmeras consequências sociais, reduzindo o acesso dos pacientes com doenças crônicas, como o diabetes mellitus a serviços de saúde, exposição a maior estresse e ansiedade e, alterações nos hábitos de vida e alimentares como demonstrado na coorte NutriNet Brasil (WHO, 2021; HUSSAIN; BHOWMIK; DO VALE MOREIRA, 2020; GARCIA et al., 2020; STEELE et al., 2020).

Desta maneira foi observado maior prevalência de depressão e de ansiedade em pessoas submetidas ao distanciamento social, quando comparadas as não afetadas por estas medidas (ETXEBARRIA et al., 2020). O convívio com situações ansiogênicas e estressantes, como perda do emprego, situações de trabalho inseguras e redução de rendimentos, pode resultar na piora dos comportamentos de saúde, resultando em descompensação clínica do DM2 (BARONE et al., 2020).

Percebendo o quanto os pacientes com DM2 demonstravam-se fragilizados durante a pandemia de Covid-19, surgiu o objetivo de revisar dentro da literatura científica como está o controle glicêmico de pessoas com Diabete Mellitus tipo II durante a pandemia da Covid-19.

\section{METODOLOGIA}

Neste capítulo será apresentado a descrição da metodologia de pesquisa aplicada na realização do presente estudo. De acordo com Gerhardt e Silveira (2009), a metodologia está atrelada a escolha teórica a ser utilizada pelo executor da pesquisa, objetivando coordenar o objetivo do estudo, natureza, procedimentos técnicos, entre outros.

Este estudo trata-se de uma revisão integrativa da literatura, a qual permite potencializar a busca de resultados de uma temática de maneira facilmente reprodutível, sendo um método característico, que relata o passado da literatura empírica ou teórica, para fornecer uma compreensão holística de um fenômeno específico (BROOME, 2006).

A busca foi delimitada a artigos de cunho científico publicados a partir de janeiro de 2020 até agosto de 2021 e indexados junto a base eletrônica PubMed, Scielo, 
Science Direct e, que avaliassem o controle glicêmico por parte de pessoas com DM2 durante a pandemia de Covid-19.

Em etapa que antecedeu a busca realizada as bases científicas foram definidos os descritores utilizados, com auxílio do Portal DeCS (descritores em Ciências da Saúde). Os seguintes descritores foram utilizados: Diabetes tipo 2, controle glicêmico, Covid-19 e Sars-CoV-2, HbA1c e suas respectivas versões em inglês. Também foram utilizados os operadores booleanos "OR" para selecionar artigos com o descritor Covid-19 ou Sars-CoV-2 e "AND" para os demais descritores.

Considerando o método e descritores mencionados, foram encontrados inicialmente 90 artigos, destes, excluímos todos os artigos que não continham a palavra "controle glicêmico" ou "glycemic control" no título, ou que avaliassem pessoas que tiveram Covid-19, devido ao Sars-CoV-2 agredir células pancreáticas e elevando a glicemia, o que alteraria o controle glicêmico independentemente dos demais fatores. Também foram excluídos estudos que não fossem realizados exclusivamente com maiores de 18 anos, estivessem avaliando apenas o método de controle glicêmico intermitente ou contínuo ou ainda que não fossem relacionados ao DM2.

A identificação e a seleção dos artigos foram realizadas por dois pesquisadores de forma independente, os quais selecionaram inicialmente os estudos pelos títulos e posteriormente pelos resumos obtidos. Após seleção inicial, realizou-se uma nova análise mais criteriosa de todas as publicações, e de forma consensual, foram determinados os estudos a serem incluídos. O período de busca nas bases eletrônicas foi durante o mês de agosto de 2021. Ao final desta etapa foram selecionados 7 estudos, conforme Tabela 1.

\section{RESULTADOS E DISCUSSÕES}

As mudanças de comportamento nos hábitos de vida e atividade física, assim como o aumento nos níveis de ansiedade e estresse são fatores que influenciam no autocuidado com o diabetes e controle glicêmico (Ruissen et al., 2021). Segundo a Organização Mundial da Saúde (2020), pessoas com diabetes têm risco mais elevado de desenvolver quadros graves da COVID-19. Sabemos que o mau controle do diabetes e o aumento da glicemia estão associados ao aumento da mortalidade 
Tabela 1: Descrição dos artigos selecionados com título, ano de publicação, autores e breve resumo da metodologia.

\begin{tabular}{|c|c|c|c|}
\hline Artigo & Ano & Autores & Metodologia \\
\hline $\begin{array}{l}\text { Efect of coronavirus disease } 2019 \text { pandemic on the } \\
\text { lifestyle and glycemic control in patients with type } 2 \\
\text { diabetes: a cross-section and retrospective cohort study }\end{array}$ & 2021 & $\begin{array}{c}\text { Chihiro Munekawa } \\
\text { e colaboradores }\end{array}$ & $\begin{array}{l}\text { Estudo de coorte transversal e retrospectivo, } \\
\text { através de aplicação de questionário } \\
\text { direcionado a pacientes com DM2, no } \\
\text { período de } 16 \text { de abril a } 1 \text { de maio de } 2020 \text {. } \\
\text { Estudo com } 183 \text { participantes. }\end{array}$ \\
\hline
\end{tabular}

Estudo observacional retrospectivo baseado em prontuários de pacientes com DM2.

Weight change and glycemic control in type 2 diabetes patients during COVID-19 pandemic: the lockdown effect

2021 Emilia Biamonte $e$ colaboradores

Realizado em dois periodos dez/2019 a maio/2020 e na retomada das atividades clínicas de maio/2020 a junho/2020. Estudo com 128 participantes.

\begin{tabular}{|c|c|c|c|}
\hline $\begin{array}{l}\text { Increased stress, weight gain and less exercise in } \\
\text { relation to glycemic control in people with type } 1 \text { and type } \\
2 \text { diabetes during the COVID-19 pandemic }\end{array}$ & 2021 & $\begin{array}{l}\text { Merel M Ruissen e } \\
\text { colaboradores }\end{array}$ & $\begin{array}{c}\text { Estudo de coorte observacional de curto } \\
\text { prazo realizado através da aplicação de } \\
\text { questionário online a individuos com DM1 e } \\
\text { DM2 durante } 8-11 \text { semanas a partir de } \\
\text { março/2020, sendo mantido lockdown } \\
\text { durante todo o periodo. Estudo com } 435 \\
\text { participantes. }\end{array}$ \\
\hline $\begin{array}{l}\text { Effects of the COVID-19 lockdown on glycaemic control } \\
\text { in subjects with type } 2 \text { diabetes: the glycalock study }\end{array}$ & 2021 & $\begin{array}{l}\text { Luca D'Onofrio e } \\
\text { colaboradores }\end{array}$ & $\begin{array}{l}\text { Este foi um estudo observacional } \\
\text { retrospectivo realizado com pacientes DM2, } \\
\text { avaliados antes ( } 3 \text { meses até dez/2019) e } \\
\text { após ( } 1 \text { mês desde } 06 / 2020 \text { ) lokcdown. } \\
\text { Estudo com } 264 \text { participantes. }\end{array}$ \\
\hline $\begin{array}{l}\text { The effect of COVID-19 lockdown on glycemic control in } \\
\text { patients with type } 2 \text { diabetes mellitus in Turkey }\end{array}$ & 2020 & $\begin{array}{l}\text { Attila Onmez e } \\
\text { colaboradores }\end{array}$ & $\begin{array}{c}\text { Estudo retrospectivo e observacional em } \\
\text { pacientes com DM2, foram avaliados dados } \\
\text { clínicos e comportamentais nas consultas } \\
\text { após lockdown de } 75 \text { dias entre março de } \\
\text { junho de } 2020 \text {, avaliando os dados } \\
\text { retrospectivamente. Estudo com } 101 \\
\text { participantes. }\end{array}$ \\
\hline $\begin{array}{l}\text { Impact of COVID-19 pandemic on glycemic control } \\
\text { among outpatients with type } 2 \text { diabetes in Japan: A } \\
\text { hospital-based survey from a country without lockdown }\end{array}$ & 2021 & $\begin{array}{l}\text { Yasuhiro Tanji e } \\
\text { colaboradores }\end{array}$ & $\begin{array}{l}\text { Estudo observacional de coorte histórica, } \\
\text { baseado na revisão de prontuários } \\
\text { eletrônicos de pacientes com DM2 no } \\
\text { período de janeiro de } 2019 \text { a gosto de } 2020 . \\
\text { Estudo com } 1009 \text { participantes. }\end{array}$ \\
\hline $\begin{array}{l}\text { Effects of COVID-19 lockdown on type } 2 \text { diabetes, } \\
\text { lifestyle and psychosocial health: A hospital-based cross- } \\
\text { sectional survey from South India }\end{array}$ & 2020 & $\begin{array}{l}\text { Prasanth Sankar e } \\
\text { colaboradores }\end{array}$ & $\begin{array}{l}\text { Foram avaliados pacientes DM2 através de } \\
\text { entrevista, periodo pós lockdown e consultas } \\
\text { a prontuário eletrônico para pré e pós } \\
\text { lockdown, coletando informações referentes } \\
\text { a estilo de vida, fatores psicossociais, uso } \\
\text { de tecnologia e dados bioquimicos. Estudo } \\
\text { com } 110 \text { participantes. }\end{array}$ \\
\hline
\end{tabular}

em pacientes com COVID-19 (GENTILE et al., 2020; ZHOU et al., 2020).

Ruissen et al. (2021) avaliaram na Holanda se o lockdown afetou o controle de peso corporal, nível de atividade física e estresse para o controle glicêmico em pessoas com DM1 e DM2 através de um questionário que analisou os hábitos diários, exercício físico e nível de estresse psicológico, através da "Escala de Estresse Percebido", além dos fármacos utilizados no tratamento e hemoglobina glicada (HbA1b). Foi encontrada associação na percepção do estresse e nível de HbA1c nas pessoas que relataram maior dificuldade no controle glicêmico devido ao maior nível 
de estresse durante o período de lockdown. Pessoas com DM1 tiveram mais foco no automonitoramento dos níveis de glicemia e, apesar dessas mudanças, nenhuma deterioração significativa no controle glicêmico foi observada.

Em outro estudo realizado com grande número de participantes no Japão, Yasuhiro Tanji et al. (2021) analisaram o controle da glicemia de 1009 pacientes com diabetes durante o decreto de estado de emergência da COVID-19, mesmo sem adotar o lockdown no país. De acordo com os dados fornecidos no prontuário eletrônico de uma clínica no Japão, os pesquisadores observaram uma piora significativa nos valores de Hba1c em pacientes DM2. O pior controle glicêmico foi observado em mulheres, pessoas com $\geq 65$, e com IMC $\geq 25$ e que não utilizavam insulina.

Chihiro Munekawa et al., (2021) também concluíram que o fator de estresse teve grande influência na diminuição do nível de exercício físico diário, e a ligação direta, com o aumento do peso corporal e HbA1c. Esse estudo de coorte transversal e retrospectivo realizado no Japão, utilizou um questionário para a obtenção de dados. Dentre eles, foi possível observar a significativa relação entre a redução da prática de atividade física e o aumento do consumo de lanches e alimentos ultra processados, resultando na alteração do peso corporal em indivíduos com idade superior a 65 anos. Ainda, ressaltou-se a importância em incentivar a seguir bons hábitos alimentares e a realização de atividade física regular.

Em uma pesquisa desenvolvida na região de Lazio, na Itália, Luca D'Onofrio et al. (2021) observaram dois públicos distintos, os quais 141 eram pacientes com DM2 expostos a pandemia causada pelo COVID-19, e a análise de dados de 123 pacientes com DM2 que frequentaram os centros de estudo no ano anterior. Foi realizada da avaliação clínica e bioquímica associadas a aplicação de um questionário de autopercepção da saúde mental no grupo exposto ao período de pandemia. A partir dessa análise notou-se que a queda do bem-estar psicológico interfere negativamente no controle do estado metabólico de HbA1c. Pacientes aposentados obtiveram uma melhora nos níveis de HbA1c durante o lockdown (pré-lockdown HbA1c 7,4\% [6,8\% 8,1\%] vs. pós-lockdown $\mathrm{HbA} 1 \mathrm{c} 7,3 \%$ [6,4\%-7,9\%]; $\mathrm{p}=0,006)$, sugerindo que 0 aumento de tempo é um fator importante para um bom manejo do diabetes. No 
entanto, não houve uma diferença relevante no controle glicêmico em pacientes com DM2.

Outro estudo também compara o estado metabólico de 128 pacientes com DM2 no período anterior e após lockdown na Itália, Emilia Biamonte et al. basearam-se nos prontuários desse grupo. A maior parte desses pacientes encontravam-se com obesidade segundo o IMC, mas com um bom controle dos níveis de glicemia. Os pesquisadores concluíram que esse período teve um impacto importante na saúde dos participantes do estudo, especialmente nos insulinodependentes devido a alterações nos níveis de Hba1c (de $7 \pm 0,8$ a 7,3 \pm 0,9\%), após o aumento do peso (79,7 Kg para 81,4 Kg), circunferência da cintura (de 103,8 cm a 105 cm) e Índice de Massa Corporal (IMC: de 29,5 Kg/m² $\pm 6 \mathrm{~kg} / \mathrm{m} 2$ a 30,1 Kg/m² $\pm 6,3 \mathrm{~kg}$ ). Por último, salienta sobre a importância do monitoramento dos pacientes com DM2 em um longo período de lockdown.

Attila Onmez et al. avaliaram 101 pacientes com DM2 na Turquia. O estudo levantou dados antropométricos e comportamentais dos pacientes que voltaram a fazer o acompanhamento em uma clínica, e assim puderam comparar o controle glicêmico e peso corporal atual com aqueles apresentados antes da pandemia. Também foi possível avaliar o controle da frequência do teste de glicose realizado pelos participantes durante o lockdown, observou-se que 13,8\% nunca realizaram o teste, $36,6 \%$ raramente, $23,7 \%$ ocasionalmente, e 25,7\% avaliaram regularmente 0 nível de açúcar na corrente sanguínea. O aumento do peso corporal (peso médio prélockdown dos pacientes foi de $84,7 \pm 16,4 \mathrm{~kg}$, aumentando para $85,5 \pm 16,8 \mathrm{~kg}$ ), nível de glicose em jejum (de 157,9 (83-645) mg / dl para 163,2 (84-550) mg / dl) e os parâmetros de $\operatorname{HbA1c}(7,67 \pm 1,76$ para 8,11 $\pm 2,48)$ nesse estudo foram mínimos, no entanto, os autores sugerem que mais estudos sejam realizados em uma população maior.

Já em pesquisa realizada na Índia por Prasanth Sankar et al. (2020), foi observado o aumento do estresse mental em apenas $15,5 \%$ da população. Dado a isso, os hábitos de vida se mantiveram durante o lockdown, mantendo boa aderência a dieta $(80,9 \%$ relataram aumento do consumo de vegetais), e sem alterações nos níveis de exercício físico. Não havendo diferenças significativas nos parâmetros 
glicêmicos e níveis de HbA1c após lockdown (8,2 $\pm 1,3 \%)$ e antes do lockdown $(8,12$ $\pm 1,6 \%$ ), em contrapartida, o peso corporal sofreu um aumento importante quando comparado ao valor antes do lockdown $(71,5 \pm 14,8 \mathrm{~kg})$. Esse estudo foi realizado através de um questionário formulado com base em várias pesquisas de referência, e aplicado durante os acompanhamentos no hospital com os pacientes que retornaram para atendimento. Diante disso os autores concluíram que o lockdown não causou um grande impacto no controle glicêmico geral e o controle glicêmico deficiente foi percebido em pacientes que reduziram a prática de atividade física e adotaram uma dieta menos saudável.

O controle glicêmico em pacientes diabéticos é afetado negativamente pelas mudanças no estilo de vida impostas pelo lockdown (BANERJEE; CHAKRABORTY; PAL, 2020). O que foi possível verificar no presente estudo é que o aumento da percepção de estresse ocorreu em cinco dos sete estudos avaliados, destes, dois foram realizados no Japão, país que não adotou lockdown e outros dois na Itália e um na Holanda, que adotaram o lockdown como medida de contenção da disseminação do vírus SARS-CoV-2.

Enquanto as causas para a falta de controle glicêmico mais apresentadas pelos pacientes com DM são a restrição a prática de atividade física e mudanças alimentares durante o lockdown. Também apresentando grande relevância a redução do acesso a hospitais e farmácias e interrupção do tratamento devido ao estresse e ansiedade em relação ao risco de infeção (LEE et al., 2020). Convergindo com o que identificamos nos artigos trazidos neste estudo, onde identificaram o aumento na percepção de estresse, e associação direta com mudança negativa nos hábitos de vida com a piora dos hábitos alimentares, redução da prática de atividade física, aumento de peso e consequente aumento nos níveis sanguíneos de $\mathrm{HbA1c}$.

Para Luca D'Onofrio et. al. (2021) houve redução nos níveis de HbA1c entre os idosos enquanto Chihiro Munekawa - et al. (2021) e Yasuhiro Tanji et al. (2021), verificaram o oposto, identificando aumento dos níveis deste marcador nesta faixa etária.

Mesmo os dois estudos que não identificaram diferenças estatisticamente significantes para elevação dos níveis de HbA1c e aumento de peso salientaram que 
- período avaliado foi curto, os pacientes não haviam deixado de receber acompanhamento neste período e verificaram leve aumento nos níveis de $\mathrm{HbA1c}$ e de peso nos pacientes.

\section{CONCLUSÃO}

Foi possível verificar que a pandemia de Covid-19 provocou impactos no controle glicêmico de pacientes diabéticos, aumentando os níveis de $\mathrm{HbA1c}$. No entanto, o lockdown parece não ter sido determinante para o aumento da percepção de estresse e aumento dos níveis de HbA1c nas populações estudadas pelos artigos trazidos nesta pesquisa. Faltam estudos realizados no Brasil avaliando o controle glicêmico por pacientes DM2 durante a pandemia de Covid-19. Porém, salientamos que a ausência de estudos brasileiros pode estar relacionada a especificidade dos critérios de busca utilizados nesta pesquisa.

\section{REFERÊNCIAS}

AMERICAN DIABETES ASSOCIATION. 9. Pharmacologic Approaches to Glycemic Treatment: Standards of Medical Care in Diabetes-2019. Diabetes Care, [s. I.], v. 42, n. Supplement 1, p. S90-S102, 2019.

BANERJEE, M.; CHAKRABORTY, S.; PAL, R. Diabetes self-management amid COVID-19 pandemic. Diabetes and Metabolic Syndrome: Clinical Research and Reviews, [s. I.], v. 14, n. 4, p. 351-354, 2020.

BARONE, M. T. U. et al. The impact of COVID-19 on people with diabetes in Brazil. Diabetes Research and Clinical Practice, [s. I.], v. 166, n. January, p. 108304, 2020.

BIAMONTE, E. et al. Weight change and glycemic control in type 2 diabetes patients during COVID-19 pandemic: the lockdown effect. Endocrine, [s. I.], v. 72, n. 3, p. 604$610,2021$. 
BROOME, M. E. Integrative literature reviews for the development of concepts. In: RODGERS, B. L.; CASTRO, A. A. Revisão sistemática e meta-análise. 2006.

D'ONOFRIO, L. et al. Effects of the COVID-19 lockdown on glycaemic control in subjects with type 2 diabetes: the glycalock study. Diabetes, Obesity and Metabolism, [s. I.], v. 23, n. 7, p. 1624-1630, 2021.

DEFRONZO, R. A. From the Triumvirate to the Ominous Octet: A New Paradigm for the Treatment of Type 2 Diabetes Mellitus. Diabetes, [s. I.], v. 58, n. 4, p. 773-795, 2009.

ETXEBARRIA, N. O. et al. Stress, anxiety, and depression levels in the initial stage of the COVID-19 outbreak in a population sample in the northern Spain. Cadernos de Saude Publica, [s. I.], v. 36, n. 4, p. 1-9, 2020.

GARCIA, M. A. et al. Bem-estar de pacientes com diabetes durante a pandemia de covid-19. Departamento de Biomedicina - Centro Universitário das Faculdades Integradas de Ourinhos-UNIFIO/FEMM., [s. I.], p. 1-12, 2020.

GERHARDT, Tatiana Engel; SILVEIRA, Denise Tolfo. Métodos de pesquisa - Porto Alegre: Editora da UFRFG, 2009.

HUSSAIN, A.; BHOWMIK, B.; DO VALE MOREIRA, N. C. COVID-19 and diabetes: Knowledge in progress. Diabetes Research and Clinical Practice, [s. I.], v. 162, p. 108142, 2020.

IDF DIABETES ATLAS Ninth edition 2019. International Diabetes Federation 2021. https://www.diabetesatlas.org/en/resources/ [accessed 23/09/2021]. 
LEE, D. W. et al. Effet de l'atélocollagène sur la cicatrisation des réparations de la racine méniscale médiale à l'aide du point Mason-Allen modifié. Revue de Chirurgie Orthopédique et Traumatologique, [s. I.], v. 106, n. 5, p. 551, 2020.

MUNEKAWA, C. et al. Effect of coronavirus disease 2019 pandemic on the lifestyle and glycemic control in patients with type 2 diabetes: A cross-section and retrospective cohort study. Endocrine Journal, [s. I.], v. 68, n. 2, p. 201-210, 2021.

ÖNMEZ, A. et al. The effect of COVID-19 lockdown on glycemic control in patients with type 2 diabetes mellitus in Turkey. Diabetes \& Metabolic Syndrome: Clinical Research \& Reviews, [s. I.], v. 14, n. 6, p. 1963-1966, 2020.

PARK, Y. et al. Performance Comparison of Five SARS-CoV-2 Antibody Assays for Seroprevalence Studies. Annals of Laboratory Medicine, [s. I.], v. 42, n. 1, p. 71-78, 2022.

PEYROT, M. et al. Psychosocial problems and barriers to improved diabetes management: Results of the Cross-National Diabetes Attitudes, Wishes and Needs (DAWN) Study. Diabetic Medicine, [s. I.], v. 22, n. 10, p. 1379-1385, 2005.

RODBARD, H. W. et al. American Association of Clinical Endocrinologists medical guidelines for clinical practice for the management of diabetes mellitus. Endocrine practice: official journal of the American College of Endocrinology and the American Association of Clinical Endocrinologists, [s. I.], v. 13 Suppl 1, p. 1-68, 2007.

RUISSEN, M. M. et al. Increased stress, weight gain and less exercise in relation to glycemic control in people with type 1 and type 2 diabetes during the COVID-19 pandemic. BMJ Open Diabetes Research and Care, [s. I.], v. 9, n. 1, p. 1-7, 2021. 
RUSSELL, L. B.; SUH, D.; SAFFORD, M. A. Time requirements for diabetes selfmanagement: too much for many? The Journal of family practice, [s. I.], v. 54, n. 1, p. 52-56, 2005.

SANKAR, P. et al. Effects of COVID-19 lockdown on type 2 diabetes, lifestyle and psychosocial health: A hospital-based cross-sectional survey from South India. Diabetes \& Metabolic Syndrome: Clinical Research \& Reviews, [s. I.], v. 14, n. 6, p. 1815-1819, 2020.

SBD. Diretrizes da Sociedade Brasileira de Diabetes 2019-2020. Disponível em: https://www.diabetes.org.br/profissionais/images/DIRETRIZES-COMPLETA-20192020.pdf

SHAW, J. E.; SICREE, R. A.; ZIMMET, P. Z. Global estimates of the prevalence of diabetes for 2010 and 2030. Diabetes Research and Clinical Practice, [s. I.], v. 87, n. 1, p. 4-14, 2010.

STEELE, E. M. et al. Dietary changes in the NutriNet Brasil cohort during the covid-19 pandemic. Revista de Saude Publica, [s. I.], v. 54, p. 1-8, 2020.

TANJI, Y. et al. Impact of COVID-19 pandemic on glycemic control among outpatients with type 2 diabetes in Japan: A hospital-based survey from a country without lockdown. Diabetes Research and Clinical Practice, [s. I.], v. 176, n. January, p. 108840, 2021.

WORLD HEALTH ORGANIZATION - WHO. WHO Coronavirus (COVID-19) Dashboard. https://covid19.who.int/table (Acesso em: set 2021).

ZHU, N. et al. A Novel Coronavirus from Patients with Pneumonia in China, 2019. New England Journal of Medicine, [s. I.], v. 382, n. 8, p. 727-733, 2020. 